\title{
A Superheated Droplet Detector for Dark Matter Search
}

\author{
L.A. Hamel, L. Lessard, L. Rainville and V. Zacek[ \\ Groupe de Physique des Particules, Université de Montréal, Canada \\ and \\ Bhaskar Sur \\ AECL, Chalk River Laboratories, Canada
}

\begin{abstract}
We discuss the operation principle of a detector based on superheated droplets of Freon-12 and its feasibility for the search of weakly interacting cold dark matter particles. In particular we are interested in a neutralino search experiment in the mass range from 10 to $10^{4} \mathrm{GeV} / \mathrm{c}^{2}$ and with a sensitivity of better than $10^{-2}$ events $/ \mathrm{kg} / \mathrm{d}$. We show that our new proposed detector can be operated at ambient pressure and room temperature in a mode where it is exclusively sensitive to nuclear recoils like those following neutralino interactions, which allows a powerful background discrimination. An additional advantage of this technique is due to the fact that the detection material, Freon-12, is cheap and readily available in large quantities. Moreover we were able to show that piezoelectric transducers allow efficient event localization in large volumes.
\end{abstract}

\footnotetext{
${ }^{1}$ Corresponding author. Tel. $\quad+514 \quad 343 \quad 6722$ Fax. $\quad+514 \quad 343 \quad 6215$ E-mail zacekv@lps.umontreal.ca
} 


\section{Introduction}

Current models explaining the evolution of the universe and the measured slight anisotropy of the cosmic background radiation have in common that they predict an appreciable contribution of non-luminous, non-baryonic matter in the form of a mixture of relativistic, light particles and non-relativistic, massive particles (so-called Hot and Cold Dark Matter). Accelerator experiments and results from the first round of Dark Matter experiments explored up to now only a small range of masses and types of possible candidate particles. In particular they leave room for an interpretation of Cold Dark Matter in terms of weakly interacting massive particles with masses ranging from $20 \mathrm{GeV} / \mathrm{c}^{2}$ up to a few hundred $\mathrm{GeV} / \mathrm{c}^{2}$. Here a fitting candidate is the neutralino of the "minimal supersymmetric standard model" ([1], [2], [3], [4]). The cross section of these particles is expected to be of electroweak strength, with coherent or axial coupling. These particles are supposed to be concentrated in a spherical halo around our galaxy with a Maxwellian velocity distribution in the galactic frame with a mean velocity of about $300 \mathrm{~km} / \mathrm{s}$ and a local density at the solar system of about $0.3 \mathrm{GeV} / \mathrm{c}^{2} / \mathrm{cm}^{3}$. The detection reaction of neutralinos would be elastic scattering off a detector nucleus and the nuclear recoil energy would be the measurable quantity. Under the given assumptions the mean recoil energy would be

$$
<E_{R}>\approx 2 k e V M_{N}\left(G e V / c^{2}\right)\left[M_{X} /\left(M_{N}+M_{X}\right)\right]^{2}
$$

where $M_{N}$ and $M_{X}$ are the masses of the detector nucleus and of the neutralino respectively.

For all detector materials the recoil energies are expected to be smaller than 100 $\mathrm{keV}$ and depending on more detailed assumptions on the cross section the expected event rates in the range between 4 to $20 \mathrm{keV}$ are between 0.01 to 100 events $/ \mathrm{kg} / \mathrm{d}$. Therefore in order to ensure a reasonable count rate a large target mass of more than 50 to $100 \mathrm{~kg}$ is needed, especially if one wants to detect the slight annual variation in count rate of several percents due to the relative motion of the earth around the sun. The latter would be the decisive signature for the detection of Dark Matter candidates. Due to background limitations, however, current experimental sensitivities are still far too small ( $>$ factor 100) in order to reach the small interaction rates predicted. The main reason for this is the fact that present detectors are sensitive to all kinds of ionizing radiations. Therefore an extremely low level of radioactive impurities in the detector material and its surroundings is necessary, as well as powerful active and passive background rejection techniques.

In order to reduce the overall background sensitivity we suggested in [5] a detection method which is exclusively sensitive to the high ionization density of recoiling nuclei with $\mathrm{A}>10$, but which is insensitive to the much smaller specific ionization 
of $\alpha, \beta, \gamma$-radiation. As is well known from bubble chamber operation, vapor droplet formation in superheated liquids is a process of precisely this kind. But in contrast to the usual cyclic bubble chamber operation, the detection of nuclear recoils requires only moderately superheated liquids and a quasi-continuous operation becomes possible. A further advantage of this technique is its operation at ambient temperatures, thus avoiding the need of cryogenics.

In this paper we discuss systematic studies of an interesting technical realization of

Figure 1: Mean recoil energies expected in Dark Matter search experiments with detector liquids like $\mathrm{CCl}_{2} \mathrm{~F}_{2}$ (Freon-12) and $\mathrm{CF}_{3} \mathrm{Br}$ as a function of neutralino mass $M_{X}$.

this detection principle which promises to be ideally suited for the detection of supersymmetric dark matter candidates, namely detectors based on superheated freon droplets $([6],[7])$. After a general review of the detection principle, we investigate more in detail Freon-12 based detectors and discuss systematic studies concerning the response of our newly developed counters with $3 \%$ Freon loading to neutron induced recoils, as well as to $\alpha$ - particles and recoiling nuclei in ${ }^{241} \mathrm{Am}$ spiked detectors. Measuring the $\alpha$-detection efficiency and understanding the physical processes involved in the detection of these particles is of fundamental importance, since we expect one of the main background contributions to the detection of dark matter particles to be due to the recoils triggered by $\alpha$ - emitting activities in the decay chains of naturally present uranium and thorium contaminations. We describe systematic studies of the detector response as a function of operating temperature and present experimental tests on event detection and localization with piezolelectric sensors. We will show that modules with volumes as large as 221 can be efficiently read out with a position resolution better than $0.5 \mathrm{~cm}$. Finally the discussion of background sensitivity and overall efficiency will lead us to the conclusion that this new method has the potential of a substantially increased sensitivity for dark matter searches at relatively modest cost.

\section{Operation Principle}

It is generally agreed that the "heat spike" theory of Seitz describes well the process of vaporization of superheated liquids [8]. In this model it is assumed that protobubbles are created by thermal spikes of released heat on a particle track. The growth of proto-bubbles is damped by adiabatic cooling, heat conduction and the viscosity of the liquid. Only if the seed cavity reaches a critical volume of radius $R_{c}$, it is large enough to nucleate a macroscopic liquid-to-vapor phase transition. $R_{c}$ is 
defined by $R_{c}=2 \sigma(T) / \delta p$, where $\sigma(T)$ is the surface tension of the liquid-vapor interface at the operating temperature $\mathrm{T}$ and $\delta p=p_{v}(T)-p_{0}$ is the difference between the vapor pressure in the bubble and the externally applied pressure. Bubble formation is triggered if an amount of energy larger than a certain critical energy $E_{c}$ is deposited within a region of extension $L \leq 2 R_{c}$. $E_{c}$ can be calculated and is well approximated for Freon-12 by

$$
E_{c}(T)=(16 \pi / 3)\left(\sigma^{3}(T) / \delta p^{2}(T)\right)
$$

$\delta p$ measures the degree of superheat and the larger $\delta p$, the smaller is $R_{c}$ and also the less heat is required for drop vaporization. However as pointed out in [9], [10], [11] only a small fraction of the energy actually deposited $E_{\text {dep }}$ by an ionizing particle within the thermal spike length $L \leq 2 R_{c}$ can be used to form a critical bubble. Therefore an efficiency factor $\eta=E_{c} / E_{\text {min }}$ determines the energy threshold $E_{\text {min }}$ for recoil detection and $E_{\min }$ together with $L$ have to be determined experimentally. As will be shown below for Freon-12 the data suggest a value of $\eta$ between $2-6 \%$.

\section{Technical Realisation: The Superheated Droplet Detector}

For our prototype studies we used superheated droplet detectors as they are used in neutron dosimetry $([12],[13])$. In these counters 5 to $100 \mu \mathrm{m}$ diameter droplets of superheated liquids e.g. $\mathrm{CCl}_{2} \mathrm{~F}_{2}$ (Freon 12) are dispersed in an elastic, clear polymerized gel whose composition has been adjusted to obtain a uniform density, equal to the liquid freon density $\left(1.3 \mathrm{~g} / \mathrm{cm}^{3}\right)$. Under ambient temperature and pressure the droplets remain in a metastable, superheated condition. When the critical energy is deposited in a superheated freon droplet, there is a sudden phase transition during which the droplet is vaporized and expands into a bubble of freon gas of about $1 \mathrm{~mm}$ in diameter, which is contained by the gel. Such an event is accompanied by an acoustic shock wave, which can be detected with piezoelectric transducers. Counters which are commercially available have recoil detection thresholds as low as several $\mathrm{keV}$ to several tens of $\mathrm{keV}$, i.e. precisely in the range of sensitivity needed for our application and they are insensitive to $\beta$ - and $\gamma$ - radiation. Fig.[1] shows for comparison the mean recoil energies which we expect in a Dark Matter search experiment for a detector liquid like Freon-12 and another freon type, $\mathrm{CF}_{3} \mathrm{Br}$, and for neutralino masses ranging from $10 \mathrm{GeV} / \mathrm{c}^{2}$ to $10^{4} \mathrm{GeV} / \mathrm{c}^{2}$.

The devices used for our tests were obtained from Bubble Technology Industries [15]. $8.1 \mathrm{ml}$ of an elastic, polyacrylamide based polymer is contained in a transparent polycarbonate test tube. The device is equipped with a piston. Upon unscrewing 
Figure 2: Count rate as a function of operating temperature for Freon detectors with $3 \%$ loading and doped with $10 \mathrm{~Bq}$ of ${ }^{241} \mathrm{Am} \alpha$-activity.

the piston, the detector becomes sensitive at atmospheric pressure. After radiation induced phase transition the bubbles are held in place by the gel until the piston is reset and the gas bubbles are again reduced to liquid droplets. Properly recompressed, the detectors can be used over years. Standard detectors for dosimetry are loaded with about $0.4 \%$ active material. For our studies we use custom made detectors with $3 \%$ loading.

\section{Systematic Studies}

\subsection{Sensitivity of Freon-12 to Nuclear Recoils}

In order to understand the response of these detectors and in particular in order to get an estimate for the relevant quantities $E_{\min }$ and $L$, which determine the threshold for Dark Matter detection, we doped detectors with 3\% Freon-12 loading with a known $\alpha$-activity of ${ }^{241} \mathrm{Am}(10 \mathrm{~Bq})$. The ${ }^{241} \mathrm{Am}$ was introduced as a soluble salt and uniformly distributed in the polymer, but is not present in the freon itself. The measured count rate as a function of operating temperature in the range of $5^{0} \mathrm{C}$ to $45^{0} \mathrm{C}$ is shown in fig. [2].

Qualitatively the observed behaviour can be understood as follows: depending on the respective specific energy losses, the detectors are sensitive at low temperatures to recoil nuclei from Coulomb-scattering of incoming $\alpha$ 's and to the ${ }^{237} \mathrm{~Np}$ recoils with an energy of $93 \mathrm{keV}$, which follow the emission of an $\alpha$ - particle of 5.4 $\mathrm{MeV}$. The ${ }^{237} \mathrm{~Np}$-recoils, however, have a range of about $100 \mathrm{~nm}$ only and therefore cannot account for more than $10 \%$ of the observed bubble count rate in the temperature range from $20^{\circ}$ to $40^{\circ} \mathrm{C}$. At $40^{\circ}$ we observe a net increase in count rate. Here the detector is sufficiently sensitive that $\alpha$-particles themselves can trigger bubble formation.

Figure 3: Energy deposited within the thermal spike length $L$. The curves show a linear rise as long as the particle range is smaller than $L$ and a flat part given by $E_{d e p}=(d E / d x) L$. Droplet vaporization occurs for recoil energies with $E_{d e p} \geq E_{m i n}$. At $35^{0} \mathrm{C}$ the detector is sensitive only for recoiling fluorine and chlorine nuclei.

For a more quantitative analysis we calculated ranges and stopping powers for $\alpha$-particles and recoil nuclei in Freon with the TRIM program package [16]. In 


\begin{tabular}{|l|r|r|r|r|r|r|r|r|r|}
\hline $\mathrm{T}^{0} \mathrm{C}$ & $0^{0}$ & $5^{0}$ & $10^{0}$ & $15^{0}$ & $20^{0}$ & $25^{0}$ & $30^{0}$ & $35^{0}$ & $40^{0}$ \\
\hline $\mathrm{E}_{c}[\mathrm{keV}]$ & 6.0 & 3.2 & 2.0 & 1.2 & 0.72 & 0.40 & 0.24 & 0.14 & 0.08 \\
\hline $\mathrm{R}_{c}[\mu \mathrm{m}]$ & 0.1 & 0.09 & 0.06 & 0.05 & 0.04 & 0.03 & 0.025 & 0.02 & 0.015 \\
\hline $\mathrm{E}_{\text {min }}[\mathrm{keV}]$ & 150 & 80 & 50 & 30 & 18 & 10 & 6.0 & 3.5 & 2. \\
\hline $\mathrm{E}_{\text {dep }}^{\text {ce }}[\mathrm{keV}]$ & 15 & 13 & 9 & 8 & 6 & 4.5 & 4 & 3 & 2.25 \\
\hline $\mathrm{E}_{\text {dep }}^{C l}[\mathrm{keV}]$ & 114 & 97 & 68 & 60 & 45 & 34 & 28 & 23 & 17 \\
\hline $\mathrm{E}_{\text {dep }}^{F}[\mathrm{keV}]$ & 107 & 92 & 64 & 56 & 43 & 33 & 27 & 22 & 16 \\
\hline $\mathrm{E}_{\text {dep }}^{C}[\mathrm{keV}]$ & 74 & 63 & 45 & 39 & 30 & 22 & 18 & 15 & 11 \\
\hline $\mathrm{E}_{\text {dep }}^{S}[\mathrm{keV}]$ & 18 & 18 & 18 & 18 & 16 & 12 & 11 & 8 & 6 \\
\hline
\end{tabular}

Table 1: Temperature dependence of the parameters $E_{c}, R_{c}, E_{\min }$, which are relevant for droplet vaporization in Freon 12. The critical energy and radius, $E_{c}$ and $R_{c}$ are predicted by the Seitz theory of bubble formation $([8],[9]) . \quad E_{d e p}=(d E / d x) L$ is the energy deposited along the thermal spike length $L . E_{\min }$ is the energy which a particle effectively has to deposit in the liquid within a distance $L$ in order to trigger a phase transition. $E_{m i n}$ and $L$ have to be inferred from experimental data.

particular for detector nuclei struck by an $\alpha$-particle we find $d E / d x$-values of 1137 $\mathrm{keV} / \mu \mathrm{m}, 1074 \mathrm{keV} / \mu \mathrm{m}$ and $743 \mathrm{keV} / \mu \mathrm{m}$ for chlorine, fluorine, and carbon at their maximum energies of $2.2 \mathrm{MeV}, 3.4 \mathrm{MeV}$ and $4.5 \mathrm{MeV}$, respectively. The stopping powers then slowly decrease with decreasing energy. For $5.4 \mathrm{MeV} \alpha$-particles the stopping power peaks around $550 \mathrm{keV}$ with $d E / d x=150 \mathrm{keV} / \mu \mathrm{m}$. We assume the same temperature dependence for $E_{\text {min }}$ as for $E_{c}$ and also the same temperature dependence for the thermal spike length $L$ as for the critical radius $R_{c}$. A benchmark point for fixing the scale of $E_{\text {min }}$ originates from the response of the detectors to thermal neutrons following the reaction ${ }^{35} \mathrm{Cl}(n, p){ }^{35} \mathrm{~S}(\mathrm{Q}$-value $=598 \mathrm{keV})$. The sulfur recoils with an energy of $17 \mathrm{keV}$ (the proton adds another $1 \mathrm{keV}$ ), has a short range of $45 \mathrm{~nm}$, a specific energy loss of $390 \mathrm{keV} / \mu \mathrm{m}$ and bubble formation is observed at temperatures above $19^{\circ} \mathrm{C}$ ([9], [10]). We then determine the deposited energies $E_{d e p}=(d E / d x) L$ for recoil nuclei and $\alpha$-particles by varying $L$ in the interval $0.1 R_{c} \leq L \leq 2 R_{c}$ and compare the resulting $E_{d e p}$-values with the minimum energy $E_{\min }(T)$ needed for bubble formation. As shown in tab.[1] a thermal spike length of $L_{\text {min }} \approx R_{c}$ gives a consistent description of all the data and predicts correctly the onset of sensitivity to $\mathrm{Cl}$ - and $\mathrm{F}$ - recoils between $0^{0}$ and $5^{0} \mathrm{C}$. The detector becomes sensitive to $\mathrm{C}$ - recoils above $15^{\circ} \mathrm{C}$ and direct $\alpha$ - sensitivity sets in at $40^{\circ} \mathrm{C}$.

Using the above results and knowing that the ${ }^{35} \mathrm{Cl}-,{ }^{19} \mathrm{~F}$-, and ${ }^{12} \mathrm{C}$ - nuclei recoil with energies up to $70 \mathrm{keV}$ following Dark Matter particle interaction, we can determine the respective detection thresholds as a function of temperature. Fig. [3] shows the deposited energy $E_{d e p}$ as a function of the recoil energy $E_{r e c}$ for the three freon constituents at $35^{0} \mathrm{C}$. 
The curves show a linear rise as long as the particle range is smaller than the thermal spike length $L(T)$ and a flat part given by $E_{\text {dep }}=(d E / d x) L(T)$. Since the minimum amount of energy deposition needed is $E_{\min }=3.5 \mathrm{keV}$ at this temperature, the detector is only sensitive for fluorine and chlorine, with recoil thresholds $E_{t h}=$ $7.0 \mathrm{keV}$ and $3.5 \mathrm{keV}$ respectively. The temperature dependence of the detection threshold for ${ }^{35} \mathrm{Cl}$ is summarized in fig.[4]: At $25^{0} \mathrm{C}$ a $10 \mathrm{keV}$ chlorine recoil can still be detected (but not fluorine and carbon recoils!), at $15^{\circ} \mathrm{C}$ the detector is completely insensitive to dark matter particles of any mass, but as we saw before, the detector remains sensitive to $\alpha$-induced recoils due to $\mathrm{U} / \mathrm{Th}$ - contaminations. This temperature dependence thus provides a means to discriminate background contributions.

Figure 4: Temperature dependence of detection threshold for ${ }^{35} \mathrm{Cl}$. At $15^{0} \mathrm{C}$ the detector is completely insensitive to neutralinos of any mass.

\subsection{Droplet and Bubble Diameter Distributions}

Two groups of detectors of identical composition (in terms of percentages of freon content or loading, droplet size distributions) were used in order to study droplet and bubble diameter distributions. One group of measurements was performed with our $\alpha$-emitting ${ }^{241} \mathrm{Am}$ doped detectors. The second set of detectors did not include any radioactive material. At the laboratory temperature $\left(22^{0} \mathrm{C}\right)$ the latter were exposed to an Ac-Be neutron source, and the bubbles were counted and measured. The same measurements were performed with the Am-activated bubble detectors. In both cases bubble diameters were measured using a calibrated microscope-video camera-TV monitor arrangement. Several hundred bubble diameters were measured within 15 min after bubble formation to obtain bubble diameter distributions. While making the measurements, the detectors were monitored by piezoelectric sensors to ensure that all bubbles were detected and measured. An essentially $100 \%$ counting efficiency was achieved so that no apparent distorsion of the distributions by observational biases can be expected.

\subsubsection{Neutron irradiation}

In the case of the detectors irradiated with neutrons, the size distributions $\mathrm{dN}(\mathrm{R}) / \mathrm{dR}$ appeared to be identical for all the detectors studied. In order to understand $\mathrm{dN}(\mathrm{R}) / \mathrm{dR}$, we have to know the droplet size distribution $\mathrm{dn}(\mathrm{r}) / \mathrm{dr}$ and assume a physical mechanism for transforming the neutron energy into an activation energy 
Figure 5: a) Droplet size distribution in detectors with 3\% loading. b) Distribution of Freon gas bubbles following irradiation with fast neutrons. The probability to vaporize is proportional to its volume. The hatched areas show droplets which contribute to bubbles with diameters larger $100 \mu \mathrm{m}$. c) Bubble size distribution obtained with detectors containing a $10 \mathrm{~Bq}^{241} \mathrm{Am} \alpha$-activity. The main contribution is due to $\mathrm{Cl}, \mathrm{F}$ and $\mathrm{C}$ recoils following elastic scattering of $\alpha$-particles.

for triggering the liquid-gas phase transition in the freon droplets. We measured $\mathrm{dn}(\mathrm{r}) / \mathrm{dr}$ for three different detectors of 3\% freon-12 loading and obtained the same distribution. Combining the three sets of measurements into a single distribution, it was then possible to fit a mathematical expression with good accuracy. As can be seen on fig.[5a], the droplet distribution peaks at $10 \mu \mathrm{m}$ diameters and tails off at larger values. An expression of the form

$$
d n(r) / d r=A_{0}\left(r-p_{1}\right)\left[e^{-p_{2}\left(r-p_{1}\right)^{2}}+p_{3} e^{-\left(r-p_{1}\right)}\right]
$$

appears to describe adequately the observed distribution. As described before, at any given temperature, the neutron is detected through a recoiling nucleus of $\mathrm{Cl}, \mathrm{F}$ or $\mathrm{C}$ within a freon droplet, provided the recoiling nucleus deposits a certain minimum amount of energy $\mathrm{E}_{\min }$ along a path of minimum length $L$. Given the value of $L$ (at $22{ }^{0} \mathrm{C}$ ) and the droplet size distribution, it follows that the probability for a droplet to undertake a phase transition is proportional to its volume (hence to $\mathrm{r}^{3}$ ). If we further assume that $\mathrm{R}=\beta \mathrm{r}$, it follows that

$$
d N(R) / d R=A_{1}(\beta r)^{3} d n(\beta r) / d(\beta r)
$$

where only one parameter $\beta$ (apart from an obvious amplitude parameter $\mathrm{A}_{1}$ ) is left free. It is seen from fig. [5b] that it is thus possible to obtain a very good fit to the experimental $\mathrm{dN}(\mathrm{R}) / \mathrm{dR}$ distribution for a value of $\beta=8.0$, close to the simple approximation $R=r\left[\rho_{l} / \rho_{g}\right]^{1 / 3}$, where $\rho_{l}=1.3 \mathrm{~g} / \mathrm{cm}^{3}$ is the liquid freon density and $\rho_{g}=1.25 \times 10^{-3} \mathrm{~g} / \mathrm{cm}^{3}$ is the freon gas density. Assuming these values one gets $\beta=$ 10 .

It is readily seen from the distributions that although $50 \%$ of the droplets have diameters smaller than $13 \mu \mathrm{m}$, these droplets contribute only to 1-2\% of the bubbles produced in the irradiation of our detectors by neutrons. The hatched areas on fig. [5a] and fig. [5b] show the droplets which contribute to the production of bubbles of diameters larger than $100 \mu \mathrm{m}$. In other words, given the energy loss values for various recoiling nuclei, and the size of the thermal spike length $L$, the neutrons essentially sample the volume distribution of the droplets, and there are not enough small droplets to make a significant contribution to the neutron detection efficiency of our bubble detectors. 


\subsubsection{Response to ${ }^{241} \mathrm{Am} \alpha$-particles}

A similar analysis was made for the bubble size distributions obtained with the detectors that contained an ${ }^{241} \mathrm{Am}$ activity. For these detectors, the droplet distribution was the same as for the neutron detectors. At the temperature of $22^{0} \mathrm{C}$ at which the measurements were performed, the detectors were insensitive to $\alpha$-particles, but sensitive to $\mathrm{Cl}, \mathrm{F}, \mathrm{C}$ recoils following Coulomb scattering with $\alpha$-particles and to the ${ }^{237} \mathrm{~Np}$ recoils from the ${ }^{241} \mathrm{Am}$ decay itself. Because of the very short range of the ${ }^{237} \mathrm{~Np}$ recoils, only $\alpha$-decays occurring in a very thin shell near the surface of the droplets can trigger bubble formation, and the bubble-size distribution expected for this mechanism would be of the form $d N(R) / d R=A_{2}(\beta r)^{2} d n(\beta r) / d(\beta r)$, but due to the short range of the recoils this "surface" contribution has a negligible effect on the overall bubble diameter distribution.

Figure 6: Explosive droplet vaporization is accompanied by an acoustic signal which can be recorded by piezoelectric transducers. All bubble signals start off with a sharp negative spike lasting about $2 \mu$ sec.

The ${ }^{35} \mathrm{Cl}-,{ }^{19} \mathrm{~F}-,{ }^{12} \mathrm{C}$ - recoils give the main contribution to droplet evaporation at $22^{0} \mathrm{C}$. Given the $\alpha$-particle range in the gel or liquid freon $(50-60 \mu \mathrm{m})$, at the (relatively) low temperature used for the measurements, two different bubble size regimes can be identified. For droplet sizes much smaller than the $\alpha$-range (remember that $50 \%$ of the droplets have diameters smaller than $13 \mu \mathrm{m})$, the $\alpha$-particles essentially reach through the entire droplet volume. One should therefore expect a $R^{3} d n(R) / d R$ form to yield a good description of the bubble distribution for small diameter values. For larger R-values, on the other hand, at relatively low temperatures (and large critical energy values), only the more energetic recoils will trigger bubble formation, so that one expects the active recoils to be concentrated in a more or less thick shell near the droplet surface. This is well illustrated in fig.[5c] where an $R^{2} d n(R) / d R$ expression best fits the bubble size distribution above the maximum, and an $R^{3} d n(R) d R$ curve does better for smaller diameter values. Here again, the small droplet contribution is strongly suppressed because of the relatively long range of the $\alpha$-particles.

\subsection{Piezoelectric Signal Detection and Event Localization}

In detectors with more than $3 \%$ loading the elastic medium becomes opalescent, nontransparent and eventually optical detection of the bubbles becomes impossible. For this reason and also in order to be able to trigger electronically on the events we investigated acoustic event detection of bubble formation. Piezoelectric sensors had 
Figure 7: Pulseheight distributions recorded in a coincidence experiment with two piezoelectric sensors. A close sensor is mounted at a fixed distance $2 \mathrm{~cm}$ from a detector test tube and a far detector is moved up to $20 \mathrm{~cm}$ apart. The amplifier noise is $3 \mathrm{mV}$ (rms).

already been shown to be sensitive to the sound created by a vaporizing droplet [12. In order to simulate a larger detector volume several detector test tubes were immersed in a $(28 \times 28 \times 28) \mathrm{cm}^{3}$ water tank, with four piezoelectric sensors installed at the tank walls in such a way that the transducer surface made direct contact with the water. We use wide band sensors (CANDED type-WD) with a sensitivity bandwidth ranging from 100 to $1000 \mathrm{kHz}$. Their signals are fed into low noise current sensitive preamplifiers LM6365 with a gain of $15 \mathrm{~V} / \mu \mathrm{A}$ and a noise characteristic of $1.5 \mathrm{pA} / \sqrt{\mathrm{Hz}}$. The signals from bubble formation are very characteristic (fig. [6]) and easily distinguishible from background signals: all bubble signals start off with a sharp negative spike lasting about $2 \mu$ sec; after this, strong oscillations at about $25 \mathrm{kHz}$ arise and the amplitudes of these oscillations are then modulated with much slower $(\mathrm{kHz})$ frequencies, which vary from event to event and are probably due to sound reflections in the container.

Fig. [7] shows the pulse height distributions recorded in a coincidence experiment with two sensors, a close sensor at a fixed distance of $2 \mathrm{~cm}$ from a detector test tube and a far sensor at distances varying from $2 \mathrm{~cm}$ up to $20 \mathrm{~cm}$. At the far $20 \mathrm{~cm}$ position the signals are still clearly separated from the $3 \mathrm{mV}$ rms noise band and we find $100 \%$ efficiency to detect bubble formation. A video recording allowed correlating the electronic signals to individual bubbles in the (low loading) detectors; under these condirions full agreement was found, i.e. whenever a bubble was detectable visually, also an electronic signal was observed and vice versa.

From this, together with the observed bubble size distribution discussed in the previous section, we can infer a lower cut-off in the droplet size distribution at around $15 \mu \mathrm{m}$. Since most of the detector mass is concentrated in larger size droplets, this cut-off translates into a freon volume efficiency of $95 \%$.

Our tests furthermore showed that acoustic time-of flight methods can be used to locate bubbles. With four sensors the signals were fed into a FADC system and the event position is calculated using a neural network trained for our detector geometry. These tests indicate that an event localization is possible with a resolution of $0.5 \mathrm{~cm}$ in the tank. 
Figure 8: Neutralino induced recoil spectra for coherent scattering on ${ }^{35} \mathrm{Cl}$. The assumption is made that the dark matter particles follow a Maxwellian velocity distribution in the galactic halo. For the cross section we assume coherent interaction via Higgs boson exchange, as described e.g. in [1].

\subsection{Background Considerations}

Being not directly sensitive to $\alpha, \beta$ and $\gamma$ - radiation is the big asset of our detector. Still there are several sources of background, internal and external to the detector which we have to consider:

- The superheated droplets will be sensitive to the $100 \mathrm{keV}$ nuclear recoils following the 5 to $6 \mathrm{MeV} \alpha$ - decays due to the presence of $\mathrm{U} / \mathrm{Th}$ daughters in the detector material, i.e. the freon itself. It is therefore mandatory to bring the contamination of ${ }^{235} \mathrm{U}$ and ${ }^{232} \mathrm{Th}$ down to the level of $10^{-15} \mathrm{~g} / \mathrm{g}$ in order to reach a sensitivity of the order $10^{-3} \mathrm{cts} / \mathrm{kg} / \mathrm{d}$. Since Freon-12 is a cryogenic liquid, we are confident that such a low level of contamination is attainable.

- The detector will register with high efficiency recoiling nuclei in droplets hit by $\alpha$-particles from ${ }^{235} \mathrm{U}$ and ${ }^{232} \mathrm{Th}$ decays in the polymer. An estimate for the tolerable contamination in the polymer can be obtained from the observed countrate of $0.05 \mathrm{cts} / \mathrm{s}$ in our $10 \mathrm{~Bq}^{241} \mathrm{Am}$ spiked detectors. From that we conclude that we need a U/Th purity of $2 \times 10^{-13} \mathrm{~g} / \mathrm{g}$ in the polymer. Since the elastic gel consists to a large part $(\approx 80 \%)$ of water which is available with a purity better than $5 \times 10^{-15} \mathrm{~g} / \mathrm{g} \mathrm{U} / \mathrm{Th}$ [17], a purity of the rest of the material at the order of $1 \times 10^{-12} \mathrm{~g} / \mathrm{g}$ is acceptable.

- With the detector installed in a deep underground laboratory (e.g. SNO) cosmic muon induced background is reduced to a negligible level but consideration has still to be given to the fast neutron component coming from the rock walls. With the measured flux of $3 \times 10^{-6} \mathrm{n} / \mathrm{cm}^{2} / \mathrm{s}$ a passive shielding of $1 \mathrm{~m}$ (borated) water will reduce the neutron induced countrate to a level of several $10^{-3} \mathrm{n} / \mathrm{d}$.

- Spontaneous droplet vaporization is another potential background source, which has still to be addressed. Manufacturers of droplet detectors and [7] claim that this background is essentially non-existent, but no systematic studies at the level of our sensitivity exist at the moment.

In order to assay the potential intrinsic background and to understand its origin, measurements were performed on the surface and at the location of the Sudbury Neutrino Observatory (SNO) $2000 \mathrm{~m}$ under ground with and without $20 \mathrm{~cm}$ water 
shielding. At the surface 0.5 cts/d were recorded for standard detectors with $0.4 \%$ loading; in the mine two detectors were exposed up to now and showed no counts after 80 days. The detector masses involved in these measurements were however too small to allow meaningful conclusions about the intrinsic background of the devices. Independent of these direct measurements, the detector material was also tested for radioactive contaminations at the Gran Sasso low activity counting facility ([18]). Limits of $2.2 \mathrm{~Bq} / \mathrm{kg}$ were obtained for the ${ }^{232} \mathrm{Th}$ contamination and again the measurements were compromised by the small mass of the test samples. On the other hand substantial ${ }^{134} \mathrm{Cs},{ }^{137} \mathrm{Cs}$ activities of about $0.2 \mathrm{~Bq} / \mathrm{kg}$ were found in the samples (this is due to the presence of $\mathrm{CsCl}$ salt, which is mixed into the gel in order to match the gel density to the density of liquid Freon). Although the associated $\beta$ - and $\gamma$ activities are not harmful by themselves for our application, we suspect an important contamination of $\mathrm{U} / \mathrm{Th}$ of the unpurified salt itself. This point is subject to further clarification.

\section{Detector Sensitivity and Achievable Limits for Neutralino Detection}

The relevant quantity which has to be determined in order to compare theoretical expectations to experimental data is the nuclear recoil spectrum following elastic scattering of a dark matter particle with a freon atom and the effect of the energy threshold, which in turn depends on the operating temperature of the detector. If we assume that the dark matter particles are distributed in our galaxy in a self gravitating halo with an isotropic Maxwellian velocity distribution, one can show that the recoil spectrum can be well approximated by an exponentially falling distribution of the form

$$
d R / d E \approx\left(R_{0} /<E_{R}>\right) \exp \left(-E /<E_{R}>\right) F^{2}\left(E_{R}\right)
$$

$<E_{R}>$ is the mean recoil energy already described in eq.(1) and $F\left(E_{R}\right)$ is a form factor, which describes in the case of coherent neutralino-nucleus interactions the coherence loss for non-zero momentum transfer due to the finite size of the nucleus; for light nuclei like ${ }^{35} \mathrm{Cl}$ it is close to one ([19]). $\mathrm{R}_{0}$ is the total rate in cts $/ \mathrm{kg} / \mathrm{d}$

$$
R_{0}=N_{T}\left(\rho_{X} / M_{X}\right) \sigma<v>
$$

where $N_{T}$ denotes the number of target atoms, $\rho_{X} / M_{X}$ is the number density of neutralinos, $\sigma$ the neutralino interaction cross section and $\langle v\rangle$ the relative average velocity.

For an evaluation of the cross section $\sigma$ in the Minimal Supersymmetric Standard Model (MSSM) we follow the treatment in ref. [1]. The experimentally most favorable situation occurs for a coherent interaction with the whole nucleus via Higgs boson 
Figure 9: Detection efficiency as function of neutralino mass. The effect of the temperature dependent energy thresholds on the differential recoil spectra of chlorine is apparent.

Figure 10: The total count rate for coherent scattering on ${ }^{35} \mathrm{Cl}$ shows a temperature dependent cut-off at small masses. The broken line represents the interaction rate without these detector effects.

exchange, provided the neutralino is a zino-higgsino mixture. Setting the MSSM parameters to $\mu>0, \tan \beta=8$ and with a Higgs mass of $50 \mathrm{GeV} / \mathrm{c}^{2}$ one obtains an isotropic elastic cross section of the order of

$$
\sigma_{c o h}=4.2510^{-40} A^{2}\left(M_{N} M_{X}\right)^{2} /\left(M_{N}+M_{X}\right)^{2}\left[\mathrm{~cm}^{2}\right]
$$

where $\mathrm{A}$ is the mass number of the nucleus. If the zino-higgsino mixing is small the coherent cross section is strongly suppressed by one or two orders of magnitude and spin dependent interactions might give more important contributions. For the coherent case practically only the neutralino interactions with chlorine matter, while for the spin dependent case, as for photinos, the ${ }^{19} \mathrm{~F}$ isotope with spin $1 / 2^{+}$gives the biggest contribution ([20]). Fig.[8] shows the recoil spectra for $M_{X}=10,10^{2}$ and $10^{3} \mathrm{GeV} / \mathrm{c}^{2}$ for coherent scattering on ${ }^{35} \mathrm{Cl}$.

In order to calculate total count rates and sensitivities, the effect of the temperature - dependent energy threshold on the differential recoil spectra has to be included. The resulting detection efficiency as a function of the neutralino mass is shown in fig.[9] and as is evident from the recoil spectra, smaller masses are more strongly affected. The total count rate for coherent scattering on ${ }^{35} \mathrm{Cl}$ in fig.[10] shows a temperature dependent cut-off at small masses below $\mathrm{M}_{X}=10 \mathrm{GeV} / \mathrm{c}^{2}$. The interaction rate peaks where the neutralino mass matches the mass of the target nucleus and it decreases with increasing neutralino mass. In Fig.[11] we show the behaviour of the upper limits on the cross section for a detector with a background at the level of $10^{-3} \mathrm{cts} / \mathrm{kg} / \mathrm{d}$.

\section{Conclusions and Outlook}

We discussed the use of moderately superheated liquids in the form of superheated droplet detectors for a new type of dark matter search experiment. The advantage of this method for Dark Matter detection is that the detector material is cheap, readily available and that it is easily possible to fabricate a large mass detector. From its easy operating conditions and its suitable isotopic composition $\left({ }^{19} \mathrm{~F}\right.$ is a 
Figure 11: Curve a) shows the upper limit on the neutralino cross section which can be set by a superheated droplet detector with a background at the level of $10^{-3} \mathrm{cts} / \mathrm{kg} / \mathrm{d}$. The weak cross section for a heavy Dirac neutrino is indicated by curve b). For comparison curves c) and d) give experimental limits obtained with Gedetectors. c) is the exclusion plot for weakly interacting massive particles obtained by the Neuchatel/Caltech/PSI Ge-experiment in the St. Gotthard tunnel and d) is the result of the Heidelberg-Moscow Ge-experiment in the Gran-Sasso Laboratory $[21]$.

spin- $1 / 2^{+}$isotope) $\mathrm{CCl}_{2} \mathrm{~F}_{2}$ (i.e. Freon 12) is an interesting active material. Even more attractive because of its higher mass could be $\mathrm{CF}_{3} \mathrm{Br}$. It has a similar vapor pressure curve as Freon-12 and is also non-flammable. Compared to alternative techniques our technique is insensitive to $\alpha, \beta$ and $\gamma$ radiation, and avoids the need of cryogenics. However the detector is sensitive to neutron and $\alpha$-particle induced recoils. Therefore care has to be taken to shield away neutrons and to reduce U/Th cotaminations in the Freon 12 itself.

An evaluation of the various parameters of our detector system suggests a modular approach. In particular we are considering a unit based on a cylindrical emulsion volume of $10 \mathrm{~kg}$ emulsion. With an achievable loading of $10 \%$ we thus obtain a $1 \mathrm{~kg}$ active detector module, read with an array of 6 piezoelectric sensors connected to a data acquisition system. Such a detector will represent a realistic prototype of a droplet based detector for more detailed background studies on surface and underground. At the same time it would serve as a modular unit easily augmented to obtain the large-mass system envisaged for the search for weakly interacting massive particles.

\section{Acknowledgements}

We are indepted to H. Ing and R. Noulty from BTI Bubble Technology Industries for many helpful discussions and for providing us with various samples of specially developed superheated droplet detectors. We wish to thank C. Hargrove, Carleton University, for fruitful discussions on purification techniques. We acknowledge precious help from our collegues J.P. Martin and M. Beaulieu in data acquisition and

electronics issues. This work was supported in part by the National Research and Science Council of Canada. 


\section{References}

[1] A. Bottino et al., Phys. Lett. B295 (1992) 330

[2] K. Griest et al., Phys. Rev. D41 (1990) 3565

[3] J. McDonald et al., Phys. Lett. B283 (1992) 80

[4] M.W. Goodman and E. Witten, Phys. Rev. D41 (1990) 2388

[5] V. Zacek, Il Nuovo Cimento, 107A (1994) 291

[6] V. Zacek, Proceedings of the $2^{\text {nd }}$ Workshop on "The Dark Side of the Universe" Universita di Roma II, November 1995, Rome, Italy

[7] J.I. Collar, Phys. Rev. D54 (1996) 1247

[8] F. Seitz, Phys. Fluids 1 (1958) 2

[9] W. Lim and C.K. Wang, Nucl. Inst. and Meth. A 336 (1993) 215

[10] M.J. Harper and J.C. Rich, Nucl.Inst. and Meth. A336 (1993) 220

[11] G. Riepe and B. Hahn, Helv. phys. Acta, 34 (1961) 865

[12] R. Apfel, Nucl. Inst. and Meth. 162 (1979) 603

[13] H. Ing and B.C. Bimboim, Nucl. Tracks 8 (1984) 285

[14] J. Sawicki, Nucl. Inst. and Meth. A 336 (1993) 215

[15] BTI, Bubble Technology Industries, Chalk River, Ontario, Canada

[16] J.F. Ziegler, TRIM Version 95.4; J.F. Ziegler, J.P. Biersack and U. Littwark "The stopping Powers and Ranges of Ions in Solids", N.Y. Pergamon Press (1985)

[17] The SNO-collaboration, private communication

[18] We thank the LNGS low background counting team for their help

[19] A. Gould, Astr. Phys. Journ. 321 (1987) 571

[20] J. Ellis and R. Flores, Phys. Lett. B263 (1991) 259

[21] M. Beck, Proceedings of the Workshop on "The Dark Side of the Universe" Universita di Roma II, June 1993, Rome, Italy 